\title{
Managing diabetes and preventing complications: what makes the difference?
}

\section{Greater emphasis is needed on behavioural and psychosocial aspects in research and clinical practice}

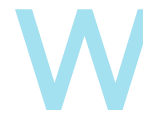

ednesday 14 November marks World Diabetes Day, the birthday of Sir Frederick Banting, whose research led to the discovery of insulin in 1922. In the 90 years since, there have been other significant landmarks. The Diabetes Control and Complications Trial (DCCT) ${ }^{1}$ and United Kingdom Prospective Diabetes Study (UKPDS) ${ }^{2}$ showed that the devastating complications of diabetes can be prevented or delayed with intensive glycaemic management. However, this has proven virtually impossible to achieve and maintain in clinical practice. In Australia, the mean glycated haemoglobin $\left(\mathrm{HbA}_{1 \mathrm{c}}\right)$ level of people with diabetes remains well above DCCT and UKPDS targets. ${ }^{3}$ This is unsurprising given the considerable challenges of managing diabetes on a daily basis: healthy eating, being physically active, taking medications as recommended, self-monitoring of blood glucose levels, all underpinned by healthy coping, problem solving, risk awareness and risk reduction. So, what makes the difference?

In Australia, new developments in diabetes focus on biomedical and technological advances. However, these raise rather than remove important behavioural and psychological issues. Indeed, the DCCT and UKPDS successes relied on their substantial but largely undocumented attention to behavioural and psychosocial factors. Thus, despite enormous public investment and considerable technological advances, the fundamental clinical and research question remains one of human behaviour: how to improve self-management of diabetes, as well as how to help doctors and health care organisations provide better support for people living with diabetes. While many individual clinicians and researchers focus on these issues, such initiatives are sporadic and unlikely to become commonplace until national policy, research funding and medical benefit schedules reflect their importance.

The World Health Organization argues that improving self-management "may have a far greater impact on the health of the population than any improvement in specific medical treatments". ${ }^{4}$ Improving such skills helps people with diabetes develop confidence to manage their condition effectively and make better use of health care consultations. Yet, few, if any, research studies have adequately characterised or measured the multifactorial processes and outcomes that could enable the widespread promotion of optimal self-care. In clinical practice, around half of Australian adults with diabetes have not been offered structured diabetes education. ${ }^{5}$ For those that have, it has usually been offered only around the time of diagnosis, despite it being unlikely that biomedical benefits will be fully sustained without further intervention.

Severe depressive symptoms affect around one in 10 Australian adults with type 1 diabetes and almost one in five with type 2 diabetes. ${ }^{5}$ This is corroborated by international systematic reviews, ${ }^{6,7}$ and the serious consequences of co-morbid depression in people with diabetes are well documented. Severe diabetes-related distress affects around one in four Australian adults with type 1 diabetes and one in five with type 2 diabetes. ${ }^{5}$ Importantly, diabetes-related distress accounts for much of the variance in depressive symptoms, indicating these symptoms may be reduced by health care professionals focusing on the psychological impact of diabetes. Unlike major depressive disorder, diabetes-related distress is significantly associated with $\mathrm{HbA}_{1 \mathrm{c}}$ level over 18 months, suggesting that reducing diabetes-related distress may improve biomedical outcomes. ${ }^{8}$ It is clear how diabetes leads to distress, with patients having to face awareness of their diagnosis, daily self-care, limitations on lifestyle, and the presence and perceived risk of diabetes-related complications. Psychologically informed approaches can significantly influence the wellbeing and health outcomes of people with diabetes. Yet, as highlighted by a recent editorial in Nature, the research funding allocated to psychological therapies is minute compared with that allocated to basic science and pharmaceutical trials. ${ }^{9}$

Furthermore, detection of major distress (general or diabetes-related) by health care professionals appears to be problematic ${ }^{10}$ unless it is systematic and structured. For almost 20 years, international guidelines have recommended routine monitoring of wellbeing, but this is not commonplace and relies on the enthusiasm of individual clinicians. Time and money is spent implementing screening programs for other complications, such as retinopathy, but not for emotional distress.

Screening for distress is relatively easy, likely to have wider benefits and may be cost-effective, but high-quality costutility studies have not been conducted. While therapists with expertise in diabetes are scarce, diabetes educators and practice nurses can be trained to identify and address most diabetes-related distress. Clinicians are often concerned that discussing the emotional impact of diabetes may open the proverbial can of worms, but it is best viewed as an opportunity to gain important insights about how an individual's diabetes self-care can be constrained by unnecessary concerns, fears, and beliefs.

Many people feel overwhelmed, frustrated, and alone with their diabetes; they want someone on their side. Consultations are not designed to enable clinicians to focus on the individual's concerns and challenges, yet doing so creates a positive, trusting relationship and identifies areas 


\section{Perspectives}

of diabetes self-care where there is most to gain. It is a myth that good communication takes more time: clinicians typically miss cues to discuss emotional concerns, and this often causes consultations to be longer than those in which clinicians recognise and act on the opportunity. ${ }^{11}$

The ideas conveyed in the recent Diabetes Australia position statement "A new language for diabetes" ${ }^{12}$ are not new — they are reinforced by two decades of research but this statement is an authoritative reminder of the powerful negative impact of inappropriate language. Clinicians reveal negative attitudes and unrealistic ideas when they describe a person with diabetes as being poorly controlled or non-compliant. While few would want to be "out of control", the idea that diabetes can truly be "controlled" is an illusion because the condition is unpredictable and difficult to manage - blood glucose levels are influenced by many factors beyond the individual's direct control (eg, hormones and stress). Nor is compliance a helpful construct, since any deviation from recommended management then allows clinicians to infer that the person with diabetes is irresponsible or uncooperative, rather than acknowledging that the advice may not suit the individual, or may not have been articulated clearly by the clinician.

Many clinicians ask how they can empower their patients to take better care of their diabetes, ignoring the fact that people with diabetes are already empowered they make a choice every time they attend (or do not attend) a consultation. The fundamental truth is that a clinician's role is not to empower. Rather, it is to not disempower (or demotivate), but we comprehensively ignore this in (continuing) medical education, and taking care not to demotivate patients is not rewarded by reimbursement systems. For people with diabetes, feeling frustrated and "like a failure" are common consequences of unrealistic expectations, and many stop confiding in their clinicians (or attending appointments) to avoid criticism. A new theory-based, practical communication strategy shown to be effective in increasing recruitment and retention in clinical trials ${ }^{13}$ is likely to prove equally useful in clinical practice for engaging distressed or ambivalent people with diabetes in their own self-care. The AASAP (anticipate, acknowledge, standardise, accept, plan) approach involves verbalising and normalising patients' concerns in a non-judgemental manner, enabling them to come to their own realistic decisions about what is best for them and their diabetes. ${ }^{13}$

The Diabetes Care Project (www.dcp.org.au) is an Australian Government initiative currently implementing and evaluating a new model of coordinated care and information technology support, providing increased funding to support those with complex comorbid conditions, and incentive payments. Ultimately, it focuses on improving $\mathrm{HbA}_{1 \mathrm{c}}$ levels. The case for monitoring wellbeing and satisfaction with care has been made to the Diabetes Care Project, but psychological measures may be used to evaluate the project's success rather than as an integral means of improving diabetes care and outcomes. There is an old adage: "what gets measured gets done". However, we need to measure what we value rather than valuing only what we measure - and, of course, if we do not measure it, we cannot know if it has changed or needs changing.

When it comes to managing diabetes and preventing complications, what makes the difference? The answer is a focus on improving self-management skills, reducing diabetes-related distress and improving clinician-patient communication - but "the evidence that behaviour is the dominant element in successful management of diabetes is so overwhelming that we tend to ignore it" ${ }^{14}$ Unless clinicians, health care organisations, policymakers and grantfunding bodies agree that the behaviour of both people with diabetes and clinicians are major contributory factors to diabetes outcomes, then we are unlikely to see this as a significant focus of research or clinical practice. World Diabetes Day offers a timely opportunity to emphasise that we need to conduct collaborative, multidisciplinary research and to establish clinical practices that value biomedical and psychological processes and outcomes equally - if we can do that, we may well achieve improvements in both.

Acknowledgements: The Australian Centre for Behavioural Research in Diabetes is a partnership for better health between Diabetes Australia - Victoria and Deakin University.

Competing interests: No relevant disclosures.

Provenance: Not commissioned; externally peer reviewed.

1 The Diabetes Control and Complications Trial Research Group. The effect of intensive treatment of diabetes on the development and progression of longterm complications in insulin-dependent diabetes mellitus. N Engl J Med 1993; 329: 683-689.

2 UK Prospective Diabetes Study Group. Intensive blood-glucose control with sulphonylureas or insulin compared with conventional treatment and risk of complications in patients with Type 2 diabetes (UKPDS 33). Lancet 1998; 352: 837-853.

3 National Association of Diabetes Centres. Australian national diabetes information audit and benchmarking (ANDIAB) 2009: final report. Canberra: Australian Government Department of Health and Ageing, 2009. http:// www.health.gov.au/internet/main/publishing.nsf/Content/pq-diabetespubs-andiab09 (accessed Nov 2012).

4 World Health Organization. Adherence to long-term therapies: evidence for action. Geneva: WHO, 2003.

5 Speight J, Browne JL, Holmes-Truscott E, et al. Diabetes MILES - Australia 2011 survey report. Canberra: Diabetes Australia, 2011. http://www.diabetesmiles .org.au/index.php/latest-news/35-diabetesmiles-australia2011surveyreport (accessed Nov 2012).

6 Ali S, Stone MA, Peters JL, et al. The prevalence of co-morbid depression in adults with Type 2 diabetes: a systematic review and meta-analysis. Diabet Med 2006; 23: 1165-1173.

7 Barnard K, Skinner T, Peveler R. The prevalence of co-morbid depression in adults with Type 1 diabetes: systematic literature review. Diabet Med 2006; 23: 445-448.

8 Fisher L, Mullan JT, Arean P, et al. Diabetes distress but not clinical depression or depressive symptoms is associated with glycemic control in both crosssectional and longitudinal analyses. Diabetes Care 2010; 33: 23-28.

9 Therapy deficit [editorial]. Nature 2012; 489: 473-474.

10 Pouwer F, Beekman ATF, Lubach C, Snoek FJ. Nurses' recognition and registration of depression, anxiety and diabetes-specific emotional problems in outpatients with diabetes mellitus. Patient Educ Couns 2006; 60: 235-240.

11 Levinson W, Gorawara-Bhat R, Lamb J. A study of patient clues and physician responses in primary care and surgical settings. JAMA 2000; 284: 1021-1027.

12 Speight J, Conn JJ, Dunning T, Skinner TC. Diabetes Australia position statement. A new language for diabetes: improving communications with and about people with diabetes. Diabetes Res Clin Pract 2012; 97: 425-531.

13 Fisher L, Hessler D, Naranjo D, Polonsky W. AASAP: a program to increase recruitment and retention in clinical trials. Patient Educ Couns 2012; 86 : 372-377.

14 Gale E. The Hawthorne studies - a fable for our times? QJM 2004; 97: 439-449. 